\title{
Visuocognitive Fluency Facilitating ECG Interpretation with Visual Metaphors and Expressive Tags
}

\author{
Jonathan R Gay \\ Ulster University, UK \\ School of Psychology \\ Cromore Road, \\ Coleraine, BT52 1SA \\ gay-j@ulster.ac.uk
}

\author{
Victoria Simms \\ Ulster University, UK \\ School of Psychology \\ Cromore Road, \\ Coleraine, BT52 1SA \\ v.simms@ulster.ac.uk
}

\author{
Raymond R Bond \\ Ulster University, UK \\ School of Computing \\ Shore Road, \\ Newtownabbey, BT37 0QB \\ rb.bond@ulster.ac.uk
}

\author{
Dewar D Finlay \\ Ulster University, UK \\ School of Engineering \\ Shore Road, \\ Newtownabbey, BT37 0QB \\ d.finlay@ulster.ac.uk
}

\author{
Helen Purchase \\ University of Glasgow, UK \\ School of Computing Science \\ 17 Lilybank Gardens \\ Glasgow, G12 8QQ \\ helen.purchase@glasgow.ac.uk
}

\author{
Aaron Peace \\ Department of Cardiology, \\ Altnagelvin Hospital, \\ Western Health and \\ Social Care Trust, UK \\ apeace@rcsi.ie
}

\author{
Stephen J Leslie \\ Cardiac Unit, \\ Ragmore Hospital, \\ Inverness, UK \\ Stephen.leslie@nhs.net
}

\begin{abstract}
The acquisition of data for medical diagnosis may be regarded as reliable and accurate, however visual representations of these data may cause significant cognitive load to novice practitioners, or learners. ECG interpretation is a case in point. This paper introduces work in progress with regard to facilitating ECG interpretation through the use and testing of visual metaphors and expressive tags. Commonly used in domains such as visual advertising, comics, musical notation, performing arts and education, visual metaphors serve as a useful heuristic to present complex concepts. Testing the potential impact of visual metaphors and expressive tags on the accuracy of ECG interpretation is novel to ECG research. The visuocognitive principles introduced in this paper have the potential to make a positive contribution to $\mathrm{HCl}$ and information visualisation.
\end{abstract}

Keywords: visuocognition, visual metaphor, expressive tags, ECG interpretation

\section{INTRODUCTION}

With the growth of computer technology in medical settings there is a potential increase of information overload for clinicians. In significantly busy work environments clinicians increasingly rely on computer representations of medical data who need accessible and effective $\mathrm{HCl}$ infovisualisations. Device recalls with usability problems are relatively common. For example, it was recently reported in User Experience Magazine that a device had to be recalled because image orientations of patients' left and right sides caused confusion and decision errors (Clark and Israelski, 2012). The visualisations were not abstract but pictorial. Conversely, abstract data output from reliable and sound devices are regularly used, yet due to their abstract visualisations information is difficult to interpret. In May 2017 the BBC reported that at least seven baby deaths at an NHS trust were deemed as avoidable. The coroner reported that the deaths related to cardiotocographs (CTG) and a contributing factor to the errors related to CTG-interpretation (Buchanan, 2017) Life and death can hinge on effective visualisations (Bond et al., 2013). A similar device to the CTG, that is regularly used worldwide, with characteristically similar abstract output and which induces a significant cognitive load is the electrocardiogram (ECG), the misinterpretation of which has led to fatalities (Breen et al., 2014). The ECG is a complex time series graph with concomitant discrete and continuous data. Its representation of cardiac electrical activity is abstract, a biosignal, with high temporal resolution. Although ECG is predominantly temporal, the electrode positional 'viewpoints' and the graph deflections provide cues to some spatial information, but for novice clinicians this information is cognitively remote and difficult to interpret (Bond et al, 2014). It is widely reported that electrocardiology is difficult to teach and learn; its concepts are complex, (Cairns et al., 2016; Zeng et al., 2015) and its 
representations are inaccessible. As such, this is a substantive and pragmatic human factors problem for clinicians (Bond et al., 2014).

\section{HUMAN FACTORS AND ECG INTERPRETATION}

ECG visual code manifests itself in twelve scattered graphs that do not correspond to the electrode 'viewpoints' thus rendering the information disparate and difficult to read. This difficulty is exacerbated when combinations of morphologies must be recognised, committed to memory, examined, and linked to cardiac pathologies to arrive at a diagnosis. Additionally, the deflections from the ECG baseline appear very similar and this lack of differentiation consequently contributes towards cognitive load and the difficulties of ECG interpretation.

Previous research shows that the use of conventional and novel visual metaphors can be a useful heuristic and a cognitive strategy that promotes cognition of complex information (Casakin, 2007; Bowdle and Gentner, 2005). Additionally, design researchers have shown that a prerequisite for cognitive fluency is the alignment of design to human visual perception (Ware, 2008). We use the term visuocognitive design to refer to infovisual design that aligns with neurocognitive features (such as metaphor comprehension and cognitive economy mechanisms) to achieve cognitive fluency. Visuocognitive design priciples may add to the Vitruvian design requirements of durability, usability and beauty (Vitruvius, circa 30BC - 15 BC; Vande Moere and Purchase, 2005). It may be argued, therefore, that visuocognitive design, visual metaphors and 'expressive tags' (as this paper coins them) may facilitate a heuristic interpretation of the ECG. An expressive tag is a graphic mark or set of marks created to express certain qualities about a graphic object or symbol. They describe four qualities: condition, motion, movement and degree. Therefore, their role is to support the intended meaning of a visual metaphor and may reduce polysemic interpretations.

\section{WORK IN PROGRESS}

Our work includes the development of a repository of pictorial schematics for the representation of ECG data for the purposes of evaluation. Drawing on Peircean visual semiotics, the schematics will use iconic, symbolic and indexical means to connote intended meaning (Table 1). The temporal data of the ECG will be spatialised over the cardiac landscape - the 'base-map' that constitutes level one of three. The second level will depict cardiac structures that have fixed positions over the base-map, and will be metaphorised using colour codes and shapes. The third level will depict unfixed cardiac pathologies and will employ visually metaphoric schematics supported by expressive tags. There will be different aspects to test. For example, the metaphoricity of a metaphor may be high or low depending on its novelty. A conventional metaphor is deeply lexicalised so little cognitive effort is needed to access its meaning (Mashal and Kasirer, 2012). However, a novel metaphor is not lexicalised, it cannot rely on previous knowledge and may need the aid of expressive tags to promote interpretation. The effect of the expressive tags needs to be investigated (Figure 1). The term 'lexicalised' is used generically here and this paper refers to the visual lexicon. As visual metaphors are different to verbal metaphors they are processed differently; pictorial information (semantic) is encoded through two neural pathways while verbal information is encoded through one route (Mashal and Kasirer, 2012). As top-down (cortical) neural processing can affect interpretation - for example, the activation of the precuneus in metaphor comprehension and semantic tasks (Mashal et al., 2014) - it is hypothesised that expressive tags will reduce polysemy, however, this hypothesis needs empirical investigation.

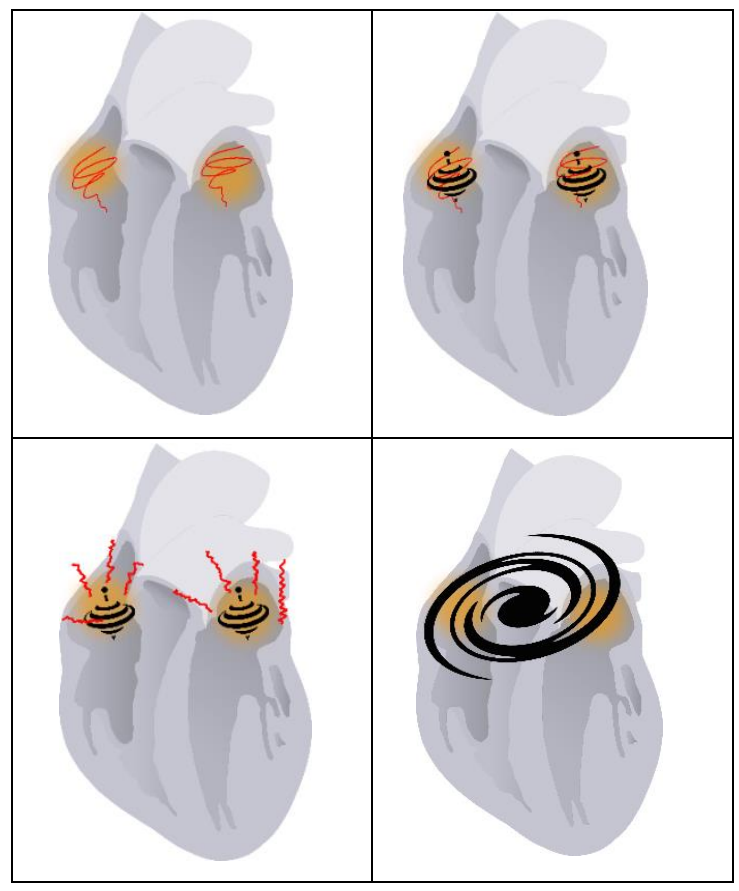

Figure 1: Options depicting Atrial Fibrillation. 
Table 1: Pictorial-schematic elements and semiotic levels. The pink box draws attention to visual metaphors, expressive tags and intuitive tags and the modes used to show cardiac pathologies. These modes include: shape, size, colour-coding, motion, movement, condition, scale, context and knowledge cues.

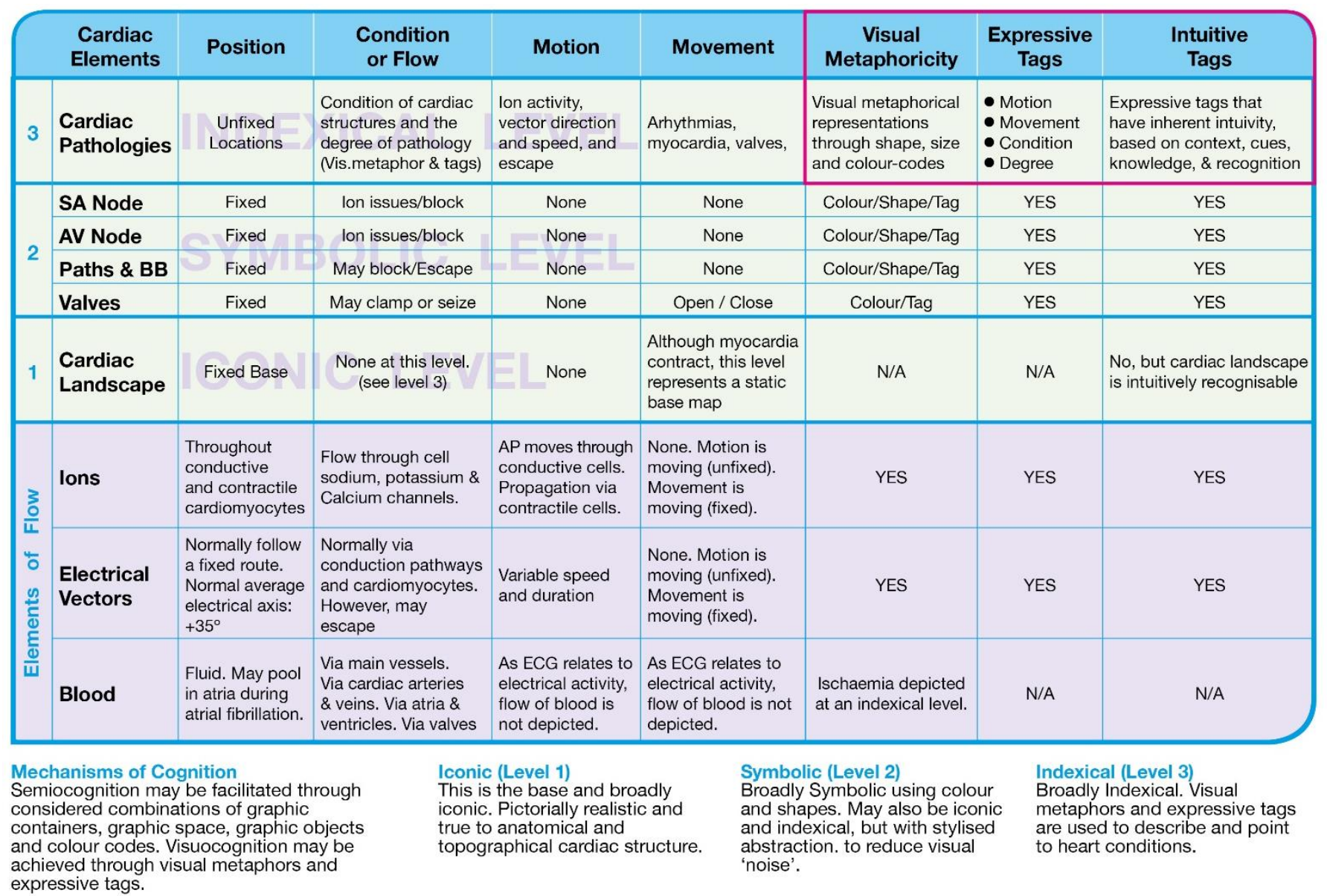

Expressive tags are like pictorial runes and can be metaphorical themselves as well as having a supportive role towards visual metaphors (Ojha, 2015). The metaphor for atrial fibrillation depicted in figure 2 is an example of this where the expressive tag (the swirl) is the metaphor. As expressive tags describe motion, movement and condition, it is probable that the right schematic achieves this more than the left schematic. The trafficlight colour codes are employed here too.

The accuracy of ECG interpretation and diagnosis is the ultimate goal of this research, thus the human factors and impact of visuocognition also need to be explored. This research makes the assumption that the technical facility to translate ECG code into accessible pictorial schematics will be in place, therefore the stimuli will be simulations.
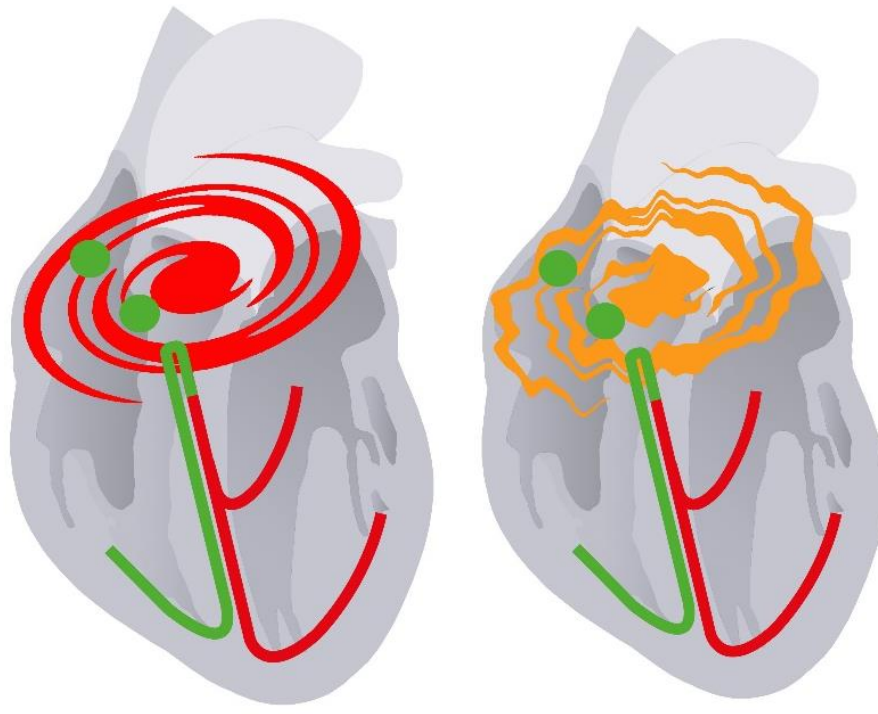

Figure 2: Atrial fibrillation with left bundle branch block. As atrial fibrillation involves repeated circular depolarisation of the atria plus the experience of rapid palpitations, the right depiction is preferable. 

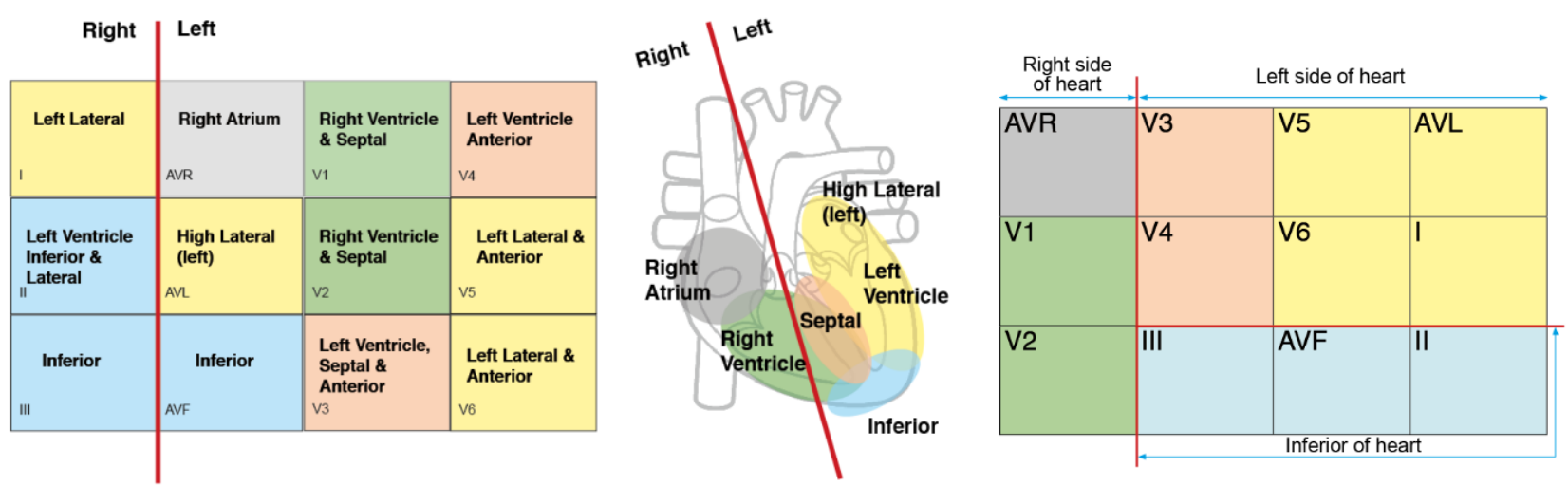

Figure 3: The central image divides the heart into right and left and labels its anatomical parts. The grid to the left represents a 12-lead ECG and, through colour coding, demonstrates that the twelve leads (or 'views') of the heart do not correspond to the coloured areas shown on the heart. The grid to the right is adjusted so that lead 'views' do correspond. For example, leads V1 and V2 'view' the right ventricle (green area), therefore they are relocated to the anatomical position - the right side of the heart.
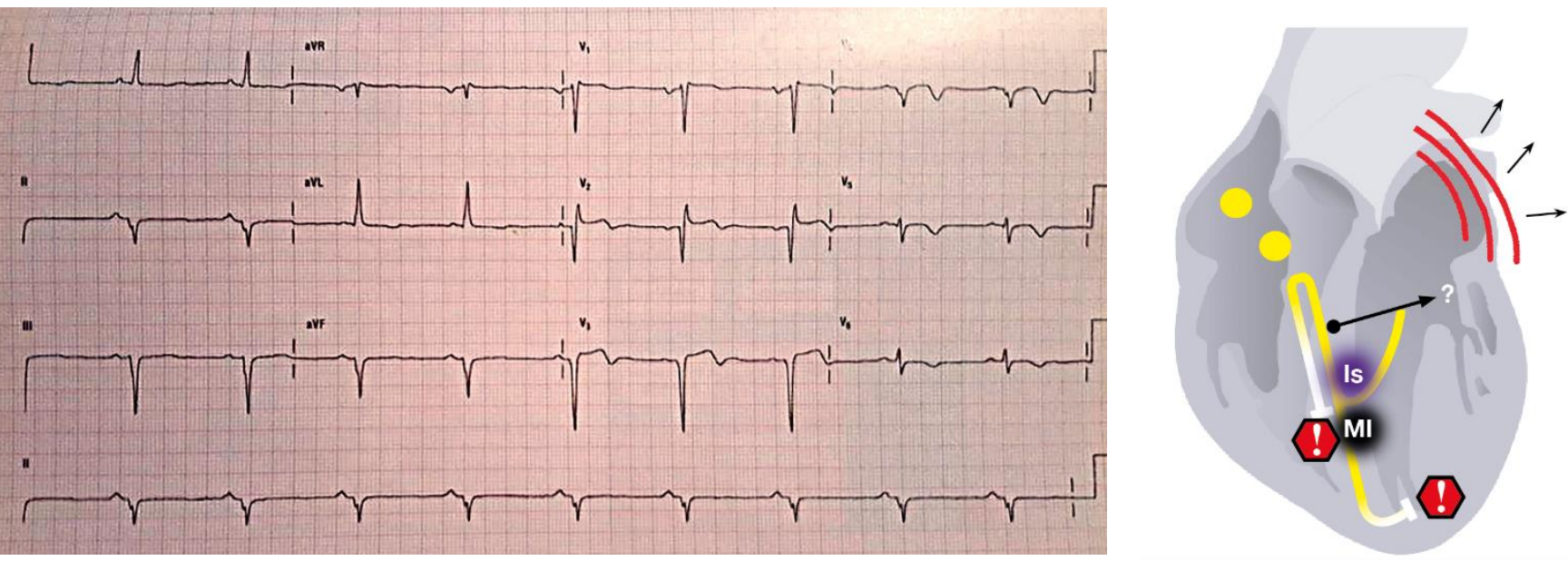

Figure 4: Morphologies such as elevated ST segments, flipped symmetrical $T$ waves and poor $R$-R progression indicate the following pathologies depicted on the pictorial schematic: an antereoseptal myocardial infarction (AMI), incomplete right bundle branch block (RBBB), left atrial enlargement ( $L A E)$, Ischaemia, and a left anterior hemiblock.

In summary, it is posited that learning electrocardiology induces cognitive load because of the following

- Lead 'views' do not correspond to $4 \times 312$ lead arrangement causing orientation issues.

- Difficulties rationalising the abstract characteristics of ECG code

- Visual similarities make differentiation of ECG anomalies difficult

- High temporal resolution and low spatial resolution make topographical orientation difficult.

- Reliance on memory rather than recognition contributes to cognitive load

- Accretions of ECG complexities evolved, absent of human factors

\section{CONCLUSION}

Claims about the complexities of ECG are well known. However, this research taxonomises them adopting a human factors and visualisation approach to test visuocognitive solutions to the problems. As such, we believe this research is novel and has scope for further investigation.

\section{Acknowledgements:}

A Peace, $R$ Bond and $S$ Leslie are supported by the European Union's INTERREG VA programme, managed by the Special EU Programmes Body (SEUPB). 


\section{REFERENCES}

Bond, R. Finlay, D, Nugent, C., and Moore, G. (2010) A Web-based tool for processing and visualizing body surface potential maps. Journal of Electrocardiology, volume 43 No. 6, pp. 560-565.

Bond, R.,Finlay, D.,Nugent, C, Moore, G. and Guldenring,D. (2013) Methods for presenting and visualising electrocardiographic data: From temporal signals to spatial imaging. Journal of Electrocardiology, volume 46 No. 3, pp 182-196.

Bond, R., Finlay, D. and Guldenring, D. (2014) Internet based ST Map Software: A Web Service, a Decision Support System and an Educational Tool. In: Computing in Cardiology, Boston, MIT. IEEE. 4.

Bond, R., Zhu, T., Finlay, D, Drew, B., Kligfield, P., Guldenring, D., Breen, C., Gallagher, A., Daly, M. and Clifford, G. (2014) Assessing computerized eye tracking technology for gaining insight into expert interpretation of the 12-lead electrocardiogram: an objective quantitative approach. Journal of Electrocardiology, volume 47 No.6. pp 895-906.

Bond, R., Finlay, D., Nugent, C., Moore, G. and Guldenring, D. (2014) A usability evaluation of medical software at an expert conference setting. Computer Methods and Programs in Biomedicine, volume 113 No.1, pp 383-395.

Bowdle, B. and Gentner, D. (2005) The Career of Metaphor. Psychological Review, volume 112, No. 1, pp193-216

Buchanan, M. (2017) Baby death cluster: Trust paid out millions in compensation, BBC4, 4 May [online]. Available at www.bbc.co.uk/news/uk-39807683

Cairns, A., Bond, R., Finlay, D., Breen, C., Guldenring, D., Gaffney, R., Gallagher, A., Peace, A., and Henn, P. (2016) A Computer-Human Interaction Model to Improve the Diagnostic Accuracy and Clinical Decision-Making during 12-lead Electrocardiogram Interpretation. Journal of Biomedical Informatics, volume 64, pp 93-107.
Cairns, A., Bond, R., Finlay, D., Guldenring, D., Badilini, F., Libretti, G., Peace, A. and Leslie, S. (2017) A Decision Support System and Rule-based Algorithm to Augment the Human Interpretation of the 12-lead Electrocardiogram. Journal of Electrocardiology, volume 50 No.6, pp 781-786.

Casakin, H. (2007) Metaphors in Design Problem Solving: Implications for Creativity. International Journal of Design, volume 1, No. 2, pp 23-35

Mashal, N. and Kasirer, A (2012) The Relationship between Visual Metaphor Comprehension and Recognition of Similarities in Children with Learning Disabilities. Research in Developmental Disabilities, volume 33, pp1741-1748

Mashal, N., Vishne, T. and Laor, N. (2014) The Role of the Precuneus in Metaphor Comprehension: Evidence from an fMRI Study in People with Schizophrenia and Healthy Participants. Frontiers in Human Neuroscience, volume 8, article 818.

Ojha, A. (2015) Visual Metaphor and Cognition. Lambert Academic Publishing

Vande Moere, A. and Purchase, H. (2005) On the Role of Design in Information Visualization. Information Visualisation, volume 10 No.4, pp356-371

Zeng, R., Yue, R., Tan, C., Wang, Q., Kuang, P., Tian, P., and Zuo, C. (2015) New Ideas for Teaching Electrocardiogram Interpretation and Improving Classroom Teaching Content. Advances in Medical Education and Practice, volume 6,pp 99104 